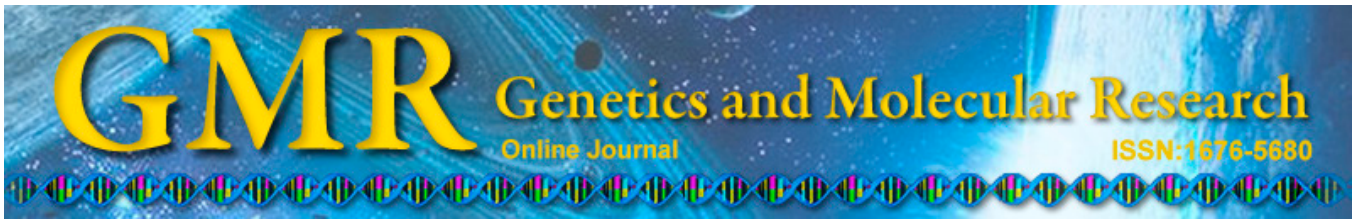

Case Report

\title{
Identification of novel compound heterozygous RECQL4 mutations and prenatal diagnosis of Baller-Gerold syndrome: a case report
}

\author{
D.H. Cao ${ }^{1 *}$, K. Mu ${ }^{2 *}$, D.N. Liu ${ }^{1}$, J.L. Sun ${ }^{1}$, X.Z. Bai ${ }^{1}$, N. Zhang ${ }^{1}$, G.B. Qiu \\ and X.W. Ma ${ }^{1}$ \\ ${ }^{1}$ Aristogenesis Center, Hospital of PLA, Shenyang, China \\ ${ }^{2}$ Genetic Disease Laboratory, Zibo Maternal and Child Health Hospital, \\ Zibo, China \\ ${ }^{3}$ Department of Laboratory Medicine, Hospital of PLA, Shenyang, China \\ *These authors contributed equally to this study. \\ Corresponding author: X.W. Ma \\ E-mail: dhcao427@sina.com
}

Genet. Mol. Res. 14 (2): 4757-4766 (2015)

Received July 8, 2014

Accepted October 29, 2014

Published May 11, 2015

DOI http://dx.doi.org/10.4238/2015.May.11.8

\begin{abstract}
Birth defects are structural and/or functional malformations present at birth that cause physical or mental disability and are important public health problems. Our study was aimed at genetic analysis and prenatal diagnosis of congenital anomalies to understand the cause of certain birth defects. Karyotypes and array-comparative genomic hybridization $(\mathrm{aCGH})$ were performed on a pregnant woman, surrounding amniotic fluid, and her husband. A short-stature panel genetic test was conducted in accordance with the phenotype of the fetus. Following examination, it was determined that the karyotype and aCGH results were normal. The RECQL4 gene in the fetus showed compound heterozygous mutations, and each parent was found to be a carrier of one of the mutations. The two heterozygous mutations (c.2059-1G>C and c.2141_2142delAG) were detected in the RECQL4 (NM_004260) gene
\end{abstract}


in the fetus; therefore, the fetus was predicted to have Baller-Gerold syndrome. These two mutations have not previously been reported. In addition, these results identified a $25 \%$ risk of the parents having a second conceptus with this congenital disease. Therefore, prenatal genetic diagnosis was highly recommended for future pregnancies.

Key words: Genotyping; Prenatal Diagnosis; Baller-Gerold syndrome; RECQL4

\section{INTRODUCTION}

Birth defects (BDs) are structural and/or functional malformations present at birth that cause physical or mental disability; some BDs may be fatal. BDs are an important public health problem due to their overall incidence, with major congenital anomalies occurring in approximately 2-3\% of live births in the European Union (EU) (Taruscio et al., 2011). The 2006 March of Dimes report estimated that, on average, 7.9 million children (approximately $6 \%$ of total births worldwide) are born with BDs (Shannon et al., 2014). However, the incidence is $5.6 \%$ in China, and there are 0.9 million new cases every year according to The Prevention Report of China's Birth Defects (2012), which was published by China's Ministry of Health. (The Central People's Government of the People's Republic of China Web Services). To control China's BD incidence, prenatal screening and diagnosis are integral to antenatal care throughout the country. Prospective parents are offered screening for common fetal chromosomal and structural congenital malformations. Prenatal screening is routinely offered in a package that includes an ultrasound scan of the fetus and an assessment of maternal blood examining biochemical markers of aneuploidy. We describe the case of a fetus with signs of Baller-Gerold syndrome (BGS) and the performance of molecular studies to assess the diagnosis of a RECQL4 mutation.

\section{MATERIAL AND METHODS}

\section{Clinical case}

The case involved a 27-year-old pregnant woman and her 31-year-old husband; there was no history of consanguinity. The patient's routine blood, urine, liver, and renal function analyses were normal. Electrocardiography and chest X-rays showed no abnormalities in the heart and lungs. The woman was 24 weeks pregnant, and the ultrasonic description was as follows: we detected bilateral arms and humerus; bilateral forearms and ulnas; bilateral thighs and femurs; bilateral legs, tibias, and fibulas; and bilateral feet. Furthermore, the left ulna was $25 \mathrm{~mm}$ long, with an "arc" shape, and no radius echo was detected; the right ulna was $24 \mathrm{~mm}$ long, and no radius echo was detected; and bilateral hands were detected in an indistinctly "hook" shape. A referral to the prenatal diagnosis clinic and chromosomal analysis were recommended. A previous patient had been pregnant with a fetus that exhibited the same ultrasonic detection patterns. Therefore, we suggested a single recessive gene disorder, and the fetus and the couple received related genetic examinations. Informed consent was obtained from the patients and the study was approved by the No. 202 Hospital of PLA Ethics Committee. All the patients consented to use their information in this study, and were aware that their 
information would be used in future research. The patient information was not anonymized. All the authors had access to any identifying information. We documented the verbal consent in their files.

\section{Karyotyping}

Karyotyping was performed on G-banded metaphases obtained from peripheral blood cultures using standard procedures. Karyotypes were obtained from the pregnant woman and her husband. Karyotyping of amniotic fluid was performed at 18 weeks of gestation according to standard techniques (Chandler and Yunis, 1978). Results are described in accordance with the International System for Chromosome Nomenclature (ISCN) 2009.

\section{Array-comparative genomic hybridization (aCGH) analysis}

Genomic DNA was extracted from peripheral blood samples (the pregnant woman and her husband) and amniocytes (fetus) using a DNA extraction kit (Tiangen Biotech; Beijing, China) according to manufacturer instructions. aCGH was performed with oligonucleotide-based custom arrays (Agilent Technologies; Santa Clara, CA, USA) using the standard protocol. Briefly, equal amounts of test DNA and normal sex-matched DNA were digested with AluI and RsaI, and differentially labeled with cyanine-5 (cy5) and cyanine-3 (Cy3) fluorescent dyes, respectively, using a SureTag Complete DNA Labeling Kit (Agilent). Hybridizations were carried out at $65^{\circ} \mathrm{C}$ for $24 \mathrm{~h}$. After washing, slides were scanned using an Agilent SureScan Microarray Scanner and the images were extracted and analyzed using the Feature Extraction v11.5 (Agilent) and the Cytogenomics v2.5 (Agilent) software, respectively.

\section{Short-stature panel genetic testing}

\section{Targeted sequence capture and next-generation sequencing}

Targeted sequence capture was performed with a BGI (Beijing Genomics Institute)developed protocol. In brief, $1 \mu \mathrm{g}$ genomic DNA was fragmented into 200-bp lengths, followed in sequence by end-repair, polyA-tailing and adaptor ligation, a 4-cycle pre-capture polymerase chain reaction (PCR) amplification, targeted sequence capture, and 15-cycle postcapture PCR. Targeted sequence capture was performed using custom oligonucleotide probe sets (NimbleGen, Roche, Basel, Switzerland). Sequencing was performed on a HiSeq2000 platform (Illumina, San Diego, California) as paired-end 90 bp (PE90) according to manufacturer instructions.

\section{Read mapping, variant detection, annotation, and interpretation}

Image analyses, error estimation, and base calling were performed using the Illumina Pipeline (version 1.3.4) to generate the primary sequence data. Low-quality reads and potential adaptor contamination were removed from the primary data using a local algorithm. The remaining short reads were mapped to the human genome (UCSC, hg18) using the BurrowsWheelerAligner (BWA) software. Single nucleotide variants (SNVs) were detected using the SOAP SNP software and small insertions and deletions (InDels) were detected using the 
SAMtools Pileup software. Variants were annotated using a BGI in-house-developed annotation pipeline and were interpreted according to the American College of Medical Genetics and Genomics (ACMG)-recommended standards (Richards et al., 2008).

\section{RESULTS}

\section{Karyotyping}

Karyotyping of G-banded metaphases showed that the karyotypes of both the couple and the fetus were normal.

\section{aCGH analysis}

We identified a copy number gain of chromosome bands $14 \mathrm{q} 32.33$ spanning approximately $0.34 \mathrm{Mb} ; 16 \mathrm{p} 11.2$ spanning approximately $1.52 \mathrm{Mb}$; and 22q11.22 spanning approximately $0.36 \mathrm{Mb}$ in non-disease associated regions. These copy number gain was determined to be polymorphism by retrieving the public database (DGV; OMIM; DECIPHER; PubMed).

\section{Targeted gene sequence capture and next-generation sequencing analysis}

\section{Targeted gene sequence capture and next-generation sequencing}

The exons and immediately adjacent intronic regions of 37 genes associated with disorders including clinical features of short limb in OMIM database (Table 1) were captured and sequenced in the proband and his healthy parents with custom oligonucleotide probe sets (NimbleGen, Roche). The targeted regions of the 37short-limbed related genes were covered by a mean depth of over 190 -fold, with $99.45 \%$ of the targeted region covered by at least one read and $97.71 \%$ by at least 20 reads.

\section{Table 1. Genes associated with short limbs Panel.}

\begin{tabular}{|c|c|c|c|}
\hline Disease name & Gene & Disease name & Gene \\
\hline Spondyloepimetaphyseal dysplasia, aggrecan type & $A C A N$ & Acromesomelic dysplasia, Hunter-Thompson type & GDF5 \\
\hline Osteogenesi simperfecta, type V & IFITM5 & Osteogenesis imperfecta, type XIII & $B M P 1$ \\
\hline Chrondrodysplasia, acromesomelic, with genital anomalies & $B M P R I B$ & Cranioectodermal dysplasia 3 & IFT43 \\
\hline Osteogenesis imperfecta, type VIII & LEPRE1 & Desbuquois dysplasia & CANT1 \\
\hline Spondyloepiphyseal dysplasia with congenital joint dislocations & CHST3 & Epiphyseal dysplasia, multiple, 5 & MATN3 \\
\hline Short rib-polydactyly syndrome, type II/II digenic & NEK1 & Osteogenesis imperfecta, types I-IV & COL1A1 \\
\hline Spondyloepimetaphyseal dysplasia, Pakistani type & PAPSS2 & Osteogenesis imperfecta, types II-IV & COL1A2 \\
\hline Spondyloepimetaphyseal dysplasia, Strudwick type & COL $2 A 1$ & Osteogenesis imperfecta, type IX & $P P I B$ \\
\hline Epiphyseal dysplasia, multiple, 6 & COL9A1 & Rothmund-Thomson syndrome & RECQL4 \\
\hline Pseudoachondroplasia & COMP & Cartilage-hair hypoplasia & $R M R P$ \\
\hline Osteogenesis imperfecta, type VII & CRTAP & Osteogenesis imperfecta, type VI & SERPINF1 \\
\hline Spondylometaphyseal dysplasia, short limb-hand type & $D D R 2$ & Osteogenesis imperfecta, type $\mathrm{X}$ & SERPINHI \\
\hline Short rib-polydactyly syndrome, type III/II digenic & $\mathrm{DYNC} 2 \mathrm{HI}$ & Epiphyseal dysplasia, multiple, 4 & $S L C 26 A 2$ \\
\hline Ellis-van Creveld syndrome & EVC & Campomelic dysplasia & SOXO \\
\hline Ellis-van Creveld syndrome & $E V C 2$ & Osteogenesis imperfecta, type XII & $S P 7$ \\
\hline Spondyloepiphyseal dysplasia tarda & TRAPPC2 & Hypochondroplasia & FGFR3 \\
\hline Short rib-polydactyly syndrome, type V & WDR35 & Osteogenesis imperfecta, type XI & FKBP10 \\
\hline Spondylocarpotarsal synostosis syndrome & $F L N B$ & & \\
\hline
\end{tabular}




\section{Identification of a potential disease-causing mutation}

More than 800 SNVs and small InDels were identified in the 37 genes analyzed in each of the three samples (Table 2). After filtering the variants with frequency more than $5 \%$ in the 1000 Genomes Database (May 2011 release; http://www.1000genomes.org/node/506) and 2\% in a BGI (Beijing Genomic Institute) in-house Database (composed of 1125 healthy control subjects) and non-functional relevance, there were approximately 86-114 non-synonymous variants (NS), splicing site (SS), or coding InDels remaining. Considering the disease inheritance pattern of genes in which these variants occur, the phenotypic overlap between the expected diseases and the proband, and the transmission pattern of the variants in the trio, the two heterozygous variants in the RECQL4 gene (c.2059-1G>C and c.2141_2142 delAG) were left as the only potential disease-causing mutations (Table 3 ). Homozygous or compound heterozygous mutations in the RECQL4 gene induce two short-limb-related syndromic autosomal recessive disorders, including Rothmund-Thomson Syndrome (RTS; OMIM: 268400) and BGS (OMIM: 218600). The c.2141_2142delAG mutation (Figure 1) transmitted from the mother induced a frameshift in exon 13 of the RECQL4 gene (the gene includes 22 exons). In addition, the c.2059-1G $>$ C mutation (Figure 2) transmitted from the father disrupted the acceptor splice site in intron 12 of the RECQL4 gene. Neither of these mutations has been previously reported in the literature, and they are expected to be the cause of the disease in the proband.

Table 2. Summary statistics on target gene coverage.

\begin{tabular}{lccc}
\hline Sample & 13S0122887 (proband) & 13B0122885 (mother) & $13 \mathrm{~B} 0122886$ (father) \\
\hline Sequence depth & 271.07 & 190.89 & 282.96 \\
$>1$ X coverage rate & $99.64 \%$ & $99.52 \%$ & $99.45 \%$ \\
$>$ 4X coverage rate & $99.49 \%$ & $99.37 \%$ & $99.18 \%$ \\
$>10 X$ coverage rate & $98.94 \%$ & $99.04 \%$ & $98.75 \%$ \\
$>$ 20X coverage rate & $98.05 \%$ & $98.5 \%$ & $97.71 \%$ \\
\hline
\end{tabular}

Table 3. Summary statistics on variants by filtering.

\begin{tabular}{lccc}
\hline Filter & Proband 13S0122887 & Mother 13B0122885 & Father 13B0122886 \\
\hline All SNVs/InDels & 5400 & 6152 & 6715 \\
NS/SS/InDel & 441 & 428 & 436 \\
$<5 \%$ variation in the 1000 Human & 56 & 74 & 59 \\
Genomes Database, and $<2 \%$ in the BGI & & - & - \\
in-house database & 2 & - & \\
Related to short upper limbs disease & 2 & & \\
\hline
\end{tabular}

$\mathrm{SNV}=$ single nucleotide variants; $\mathrm{InDel}=$ insertion or deletion mutation; $\mathrm{NS}=$ non-synonymous variant; $\mathrm{SS}=$ splice site; BGI = Beijing Genomics Institute.

\section{DISCUSSION}

The RECQL4 gene is a member of the human RecQ helicase family and plays a role in the initiation of replication. In addition, RECQL4 is implicated in several DNA repair pathways (Fan and Luo, 2008; Singh et al., 2010; Thangavel et al., 2010; Croteau et al., 2012). 


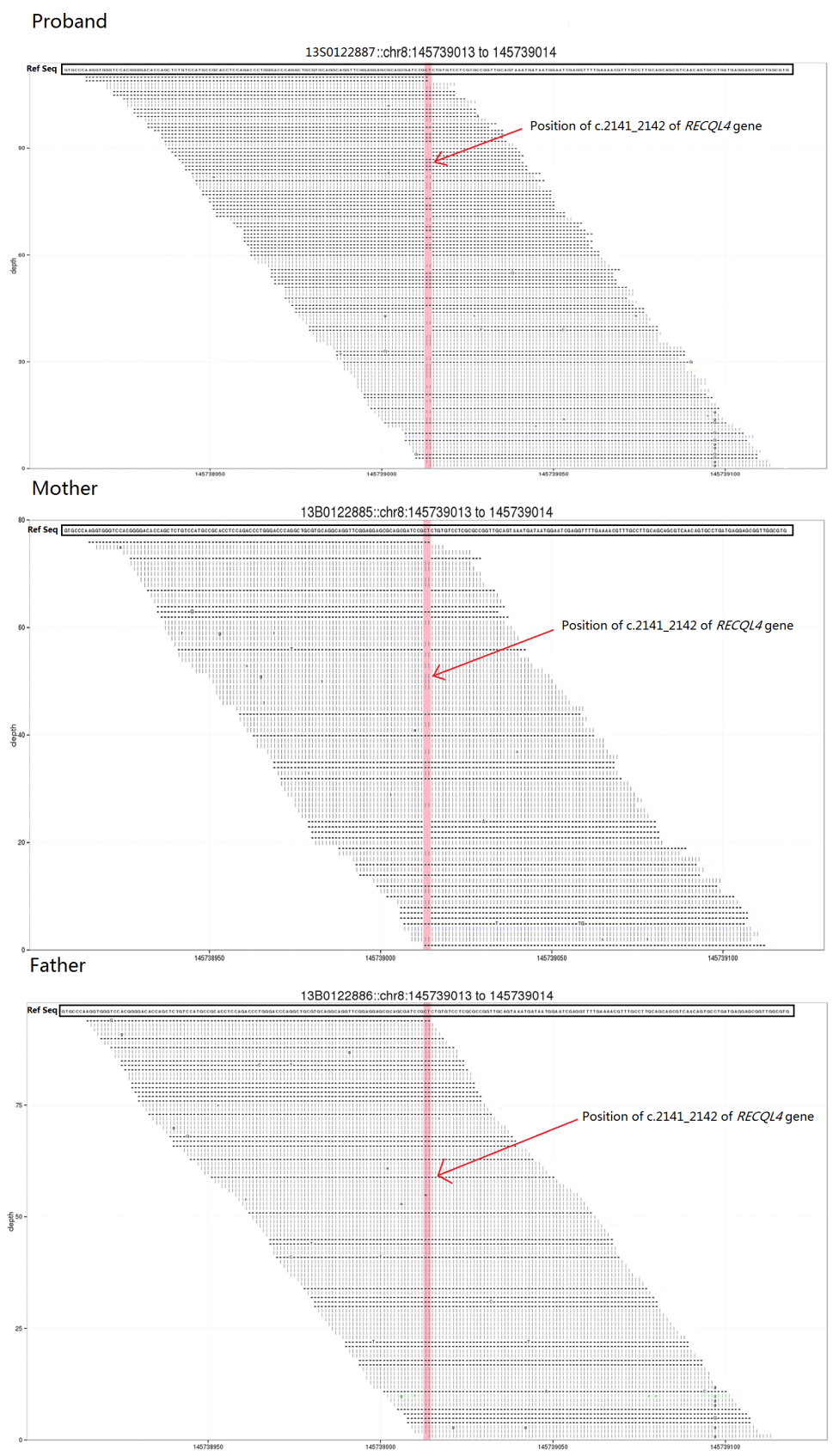

Figure 1. Sequencing reads in support of the c.2141_2142delAG mutation. The DNA sequence inside the top black box indicates reference sequence. The pink shaded box highlights the position of c.2141_2142 of RECQL4 gene. For 13B0122886 (father), no read supports c.2141_2142delAG mutation. For 13S01228 87 (proband) and 13B0122885 (mother), the support reads for c.2141_2142delAG is 45 and 44 respectively, which means this mutation is inherited from mother. 
Prenatal and genetic diagnosis of Baller-Gerold syndrome
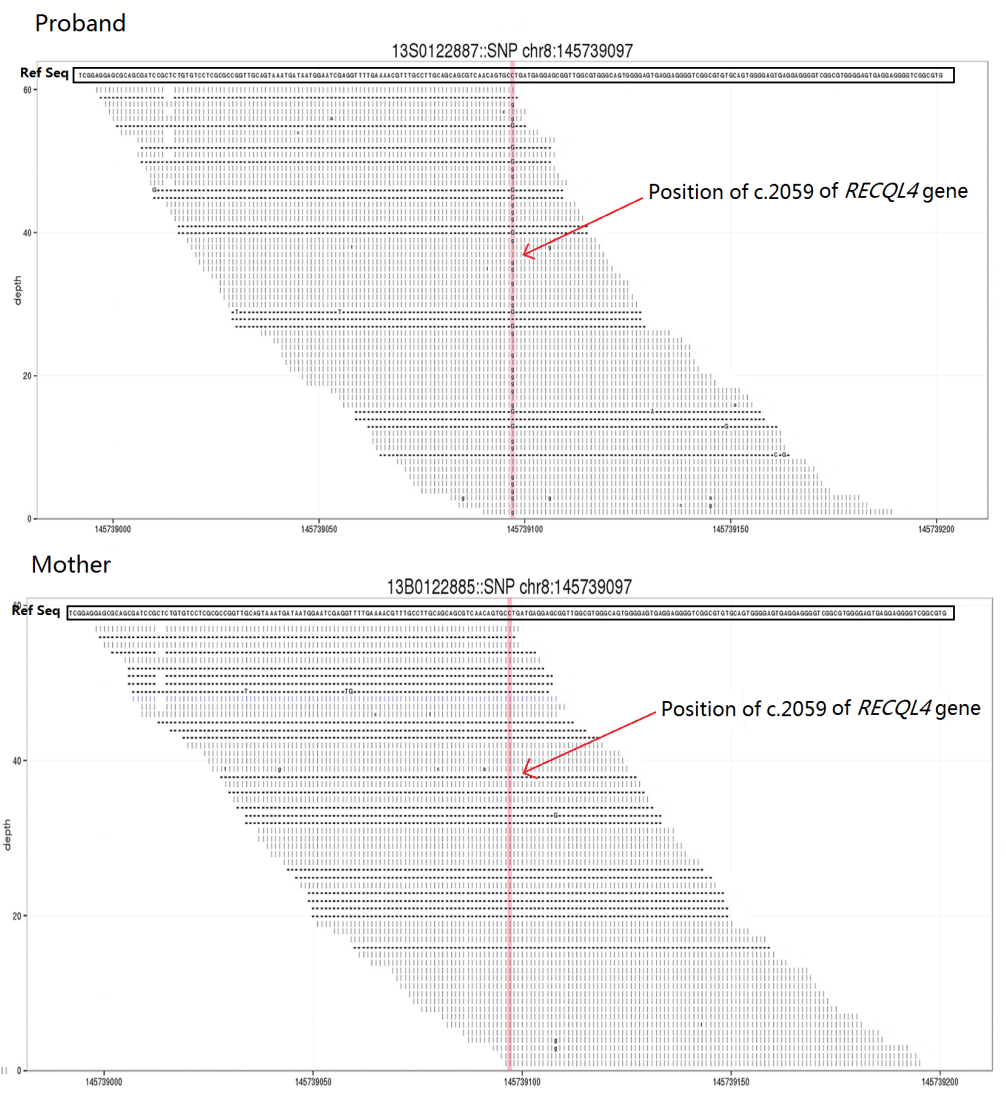

Father

13B0122886:SNP chr8:145739097

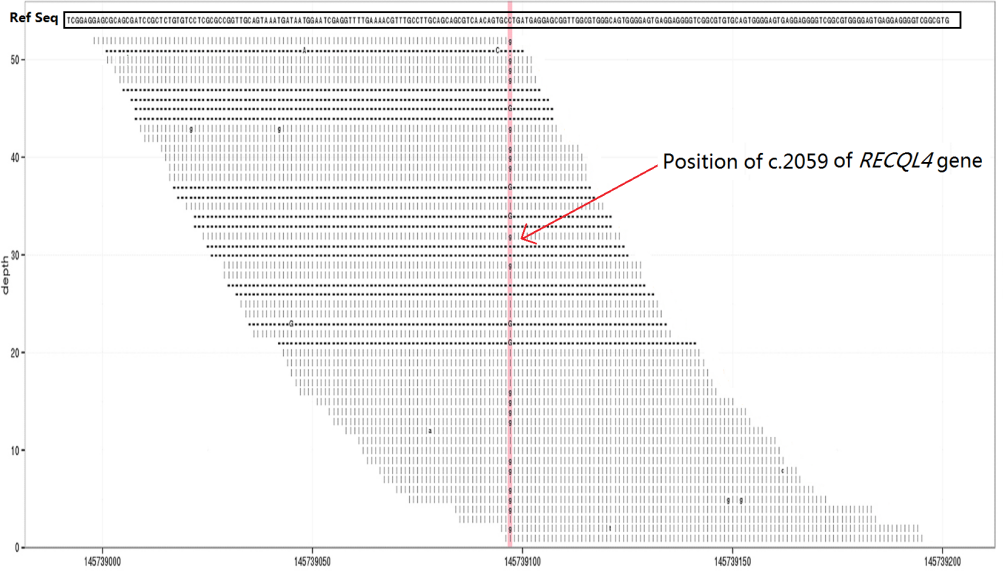

Figure 2. Sequencing reads in support of the $2059-1 \mathrm{G}>\mathrm{C}$ mutation. The DNA sequence inside the top black box indicates reference sequence. The pink shaded box highlights the position of c.2059-1 of RECQL4 gene. For 13B0122885 (mother), no read supports c.2059-1 G>C. For 13S0122887 (proband) and 13B0122886 (father), the support reads for c.2059-1 G>C is 37 and 27 respectively, which means this mutation is inherited from father. 
It was originally identified in 1998 in a human genome search for RecQ helicases (Kitao et al., 1998) and some years later was found to be associated with the rare type II RTS (Kitao et al., 1999), RAPADILINO Syndrome (Siitonen et al., 2003), and BGS (Van Maldergem et al., 2006). RTS, RAPADILINO, and BGS exhibit a significant overlap of symptoms (Capp et al., 2010), all occurring in tissues with a high proliferation rate. RTS is characterized by poikiloderma; sparse hair, eyelashes, and/or eyebrows; small stature; skeletal and dental abnormalities; cataracts; and an increased risk for cancer, especially osteosarcoma. The skin is typically normal at birth; the typical rash of RTS develops between ages three and six months as erythema, swelling, and blistering on the face and subsequently spreads to the buttocks and extremities. The rash evolves over months to years into the chronic pattern of reticulated hypo- and hyperpigmentation, punctate atrophy, and telangiectases, collectively known as poikiloderma. Hyperkeratotic lesions occur in approximately one third of individuals. Skeletal abnormalities include dysplasia, absent or malformed bones (such as absent radii), osteopenia, and delayed bone formation. The population prevalence of RTS and the carrier frequency are unknown (Larizza et al., 2012). BGS is characterized by coronal craniosynostosis manifested as abnormal shape of the skull (brachycephaly) with ocular proptosis and a bulging forehead, radial ray defect manifested as oligodactyly (reduction in number of digits), aplasia or hypoplasia of the thumb, and/or aplasia or hypoplasia of the radius, growth retardation, and poikiloderma.

In the current study, we found that the 24-week fetus exhibited a bilateral radial agenesis by ultrasound examination. In addition, bilateral thumbs were not clearly shown in the ultrasound imaging and seemed to be absent. Because this woman was pregnant with a fetus presenting the same symptoms as another fetus encountered a year and half previously, we speculated that the defects were genetic in origin. Given that the karyotype analysis and aCGH levels were normal in the blood samples of this couple and the amniotic fluid sample from the fetus, there was a high possibility that that genetic defect observed in the fetus was a single-gene disorder. Taken together, we chose to perform further genetic testing using the Short-stature Panel, and performed gene sequencing in 448 genes associated with limb development. We identified two heterozygous mutations (c.2059-1G>C and c.2141_2142delAG) in the RECQL4 (NM_004260) gene. These mutations have not been previously reported, and both were predicted to be severe loss-of-function mutations. The c.2059-1G $>$ C mutation is located in the acceptor splice site of intron 12 and is assumed to disrupt the splice site. The c.2141_2142delAG mutation is a frameshift mutation in exon 13 that leads to a premature stop at codon 807. Several nonsense mutations [p.Gln810*, p.Gln821*, p.Arg826*, p.Gln888*, and p.Gln1175* (Wang et al., 2003; Jin et al., 2008; Siitonen et al., 2009)], which occurred downstream of codon 807, have been previously reported to be the cause of RTS. The c.2059$1 \mathrm{G}>\mathrm{C}$ and c.2141 2142delAG mutations have not been observed in at least 6000 controls (1000 Genomes Project and BGI in-house database). RTS and BGS are two short-limb-related syndromes and autosomal recessive disorders. We detected the c.2059-1G>C mutation in the blood sample of the husband, and the c.2141_2142delAG mutation was detected in that of the mother; therefore, these two mutations were found to be compound heterozygous in the proband. On this basis, these mutations (c.2059-1G $>$ C and c.2141_2142delAG) in the RECQL4 gene are assumed to be the disease-causing mutations in this patient.

The pregnancy was terminated after obtaining written informed consent from the patient. The fetus aborted was found to have bilateral short upper forearms and bilateral absence of thumbs. The fetus had an identical presentation to the BGS patient previously reported (Savarirayan et al., 1998; Markose et al., 1999; Lype et al., 2008). Therefore, we believe this 
fetus had BGS. The use of the short-stature panel for genetic testing covered all the mutations, including point mutations and insertions in the exons and \pm 10 -bp neighboring introns in short-stature-associated genes. This new method of testing genes associated with similar symptoms and/or presentations in genetic disorders greatly improves the efficiency of genetic diagnosis. The identification of abnormal genes in genetic diseases can greatly aid prenatal genetic diagnosis and intervention, thus allowing the possibility of ensuring that patients are delivered of a healthy child.

\section{ACKNOWLEDGMENTS}

We thank all the patients who participated in this study. Research supported by the National Foundation of China (\#30973140), the Doctor Starting Funding of Liaoning (\#20111123), and the Natural Science Foundation of Liaoning (\#201202230).

\section{REFERENCES}

BWA software. Available at [http://sourceforge.net/projects/bio-bwa/].

Capp C, Wu JH and Hsieh, TS (2010). RecQ4: the Second Replicative Helicase? Crit. Rev. Biochem. Mol. Biol. 45: 233-242. Chandler ME and Yunis JJ (1978). A high resolution in situ hybridization technique for the direct visualization of labeled G-banded early metaphase and prophase chromosomes. Cytogenet Cell Genet. 22: 352-356.

Croteau DL, Singh DK, Hoh Ferrarelli L, Lu H, et al. (2012). RECQL4 in genomic instability and aging. Trends Genet. 28: 624-631.

DECIPHER Web Services. Available at[ http://decipher.sanger.ac.uk/].

DGV. Database of Genomic variants Web Services. Available at [http://dgv.tcag.ca/dgv/app/home].

Fan W and Luo J (2008). RecQ4 facilitates UV light-induced DNA damage repair through interaction with nucleotide excision repair factor xeroderma pigmentosum group A (XPA). J. Biol. Chem. 283: 29037-29044.

Jin W, Liu H, Zhang Y, Otta SK, et al. (2008). Sensitivity of RECQL4-deficient fibroblasts from Rothmund-Thomson syndrome patients to genotoxic agents. Hum. Genet. 123: 643-653.

Kitao S, Ohsugi I, Ichikawa K, Goto M, et al. (1998). Cloning of two new human helicase genes of the RecQ family: biological significance of multiple species in higher eukaryotes. Genomics 54: 443-452.

Kitao S, Shimamoto A, Goto M, Miller RW, et al. (1999). Mutations in RECQL4 cause a subset of cases of RothmundThomson syndrome. Nat. Genet. 22: 82-84.

Larizza L, Roversi G and Verloes A (2012). Clinical utility gene card for: Rothmund-Thomson syndrome. Eur. J. Hum. Genet. 21: doi: 10.1038/ejhg.2012.260.

Lype M, Henry P, Aravind C and Arun K (2008). Baller-Gerold syndrome: Further evidence for association with prenatal exposure to valproate. Ann. Indian Acad. Neurol. 11: 52-55.

Markose G, Fairhurst J and Temple IK (1999). A case of Baller-Gerold syndrome. Clin. Dysmorphol. 8: 69-71.

NCBI.The National Center for Biotechnology Information Web Services. Available at [http://www.ncbi.nlm.nih.gov/ books/NBK1116/?term=genereviews].

OMIM. Online Mendelian Inheritance in Man Web Services. Available at[ http://omim.org/].

Prevention Report of China's Birth Defects (2012). The Prevention Report of China's Birth Defects. Available at [http:// www.gov.cn/gzdt/2012-09/12/content_2223371.htm].

PubMed Web Services. Available at[ http://www.ncbi.nlm.nih.gov/pubmed].

Richards CS, Bale S, Bellissimo DB, Das S, et al. (2008). ACMG recommendations for standards for interpretation and reporting of sequence variations: Revisions 2007. Genet. Med. 10: 294-300.

SAMtools Pileup software. Available at [http://sourceforge.net/projects/samtools/].

Savarirayan R, Tomlinson P and Thompson E (1998). Baller-Gerold syndrome associated with congenital portal venous malformation. J. Med. Genet. 35: 767-769.

Shannon GD, Alberg C, Nacul L and Pashayan N (2014). Preconception healthcare and congenital disorders: Systematic review of the effectiveness of preconception care programs in the prevention of congenital disorders. Matern. Child Health J. 18: 1354-1379.

Siitonen HA, Kopra O, Kaariainen H, Haravuori H, et al. (2003). Molecular defect of RAPADILINO syndrome expands 
the phenotype spectrum of RECQL diseases. Hum. Mol. Genet. 12: 2837-2844.

Siitonen HA, Sotkasiira J, Biervliet M, Benmansour A, et al. (2009). The mutation spectrum in RECQL4 diseases. Eur. J. Hum. Genet. 17: 151-158.

Singh DK, Karmakar P, Aamann M, Schurman SH, et al. (2010). The involvement of human RECQL4 in DNA doublestrand break repair. Aging Cell 9: 358-371.

SOAP SNP software. Available at [http: //soap.genomics.org.cn/].

Taruscio D, Carbone P, Granata O, Baldi F, et al. (2011). Folic acid and primary prevention of birth defects. Biofactors 37: $280-284$

Thangavel S, Mendoza-Maldonado R, Tissino E, Sidorova JM, et al. (2010). Human RECQ1 and RECQ4 helicases play distinct roles in DNA replication initiation. Mol. Cell Biol. 30: 1382-1396.

The Central People's Government of the People's Republic of China Web Services. Available at[http://www.gov.cn/ gzdt/2012-09/12/content_2223371.htm].

UCSC, hg18.Web Services. Available at [http://hgdownload.cse.ucsc.edu/goldenPath/hg18].

Van Maldergem L, Siitonen HA, Jalkh N, Chouery E, et al. (2006). Revisiting the craniosynostosis-radial ray hypoplasia association: Baller-Gerold syndrome caused by mutations in the RECQL4 gene. J. Med. Genet. 43: 148-152.

Wang LL, Gannavarapu A, Kozinetz CA, Levy ML, et al. (2003). Association between osteosarcoma and deleterious mutations in the RECQL4 gene in Rothmund-Thomson syndrome. J. Natl. Cancer Inst. 95: 669-674. 